\title{
DO TWO LARGE GAPS SIGNIFY DOUBLE-POMERON EXCHANGE?
}

\author{
M.-S. CHEN and G.L. KANE \\ Physics Department, University of Michigan, Ann Arbor, Michigan 48104, USA
}

Received 31 October 1975

\begin{abstract}
We investigate in what sense the Pomeron can occur more than once in a single process by estimating the singlePomeron contribution to the double-Pomeron-exchange-like processes and comparing with the experimental data. From this comparison, we find that the double-Pomeron contribution may be much smaller than the previous theoretical estimates. Further theoretical investigation and experimental tests are suggested and discussed.
\end{abstract}

How the Pomeron can occur more than once in a single process is a crucial question in understanding the structure of the Pomeron and has received much theoretical attention in recent years $[1-3]$. This question also has led to several experimental searches [47] for the existence of the double-Pomeron exchange (DPE) in reactions of the type

$\mathrm{A}+\mathrm{B} \rightarrow \mathrm{A}+\mathrm{B}+\mathrm{X}$,

as illustrated in fig. 1a. In refs. [4-7], events in pp scattering characterized by two large rapidity gaps separating the leading particles and $X$ are observed with a cross section of approximately $20 \mu \mathrm{b}$ for $\mathrm{X}=\pi^{+} \pi^{-}$ and two units of rapidity as the minimum gap size. The crucial thing to keep in mind is that the signal of order of $20 \mu b$ is very small. That is why it is important to study the meaning of DPE, what processes could simulate it, and how to interpret the results if DPE were several times smaller than expected. Although the existence of DPE in reaction (1) implies the existence of such events, as we shall discuss, the converse is not true. Whether the results of refs. [47] are evidence for the existence of DPE should be more carefully studied. In this work, we investigate other mechanisms for producing events with two large rapidity gaps, look into the possibility of explaining the data of refs. [4-7] without the DPE, and suggest further tests of the existence of the DPE [8].

Inelastic diffractive events with a large rapidity gap separating one of the leading particles and the rest of the final state particles are observed with a cross section of approximately $4 \sim 8 \mathrm{mb}$ for pp scattering in

\footnotetext{
Work supported in part by USERDA.
}

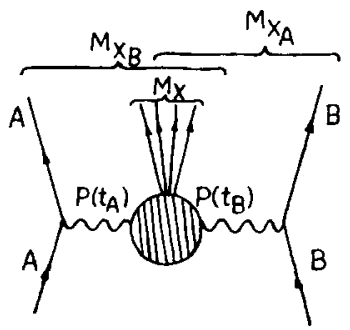

(a)

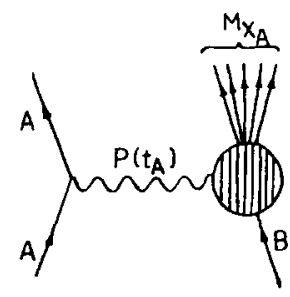

(b)
Fig. 1(a) Illustration of the double-Pomeron-exchange and the relevant variables. (b) Illustration of the inelastic single diffractive processes.

the FNAL and ISR energy range. If we assume that such inelastic processes are dominated by the Pomeron exchange, as illustrated in fig. $1 \mathrm{~b}$, the question is in what sense another Pomeron can be exchanged in the Pomeron-proton inelastic vertex to make a DPE process shown in fig. 1a. If the Pomeron is a simple factorizable pole, as in the usual scheme for the recurrance of the Pomeron, it will give rise to a two-particle inclusive cross section $[3,9,10]$

$\frac{\mathrm{d} \sigma_{\mathrm{AB}}^{\mathrm{AB}}}{\mathrm{d} t_{\mathrm{A}} \mathrm{d} Z_{\mathrm{A}} \mathrm{d} t_{\mathrm{B}} \mathrm{d} Z_{\mathrm{B}}} \approx \frac{1}{\sigma_{\mathrm{AB}}^{\text {tot }}} \frac{\mathrm{d} \sigma_{\mathrm{AB}}^{\mathrm{A}}}{\mathrm{d} t_{\mathrm{A}} \overline{\mathrm{d} Z_{\mathrm{A}}}} \frac{\mathrm{d} \sigma_{\mathrm{AB}}^{\mathrm{B}}}{\mathrm{d} t_{\mathrm{B}} \mathrm{d} Z_{\mathrm{B}}}$

for an approximately factorizable Pomeron, where

$Z_{\mathrm{A}, \mathrm{B}} \equiv \ln \left(s / M_{\mathrm{X}_{\mathrm{A}, \mathrm{B}}}^{2}\right)$,

$\sigma_{A B}^{\text {tot }}$ is the total cross section, and the differential cross sections on the right hand side of eq. (2) are the corresponding inclusive cross sections. Eq. (2) is supposed to be valid in the kinematical region with small $t_{\mathrm{A}, \mathrm{B}}$, large $Z_{\mathrm{A}, \mathrm{B}}$ and large $M_{\mathrm{X}}$. In this region, the leading 
particles are separated from the others by large rapidity gaps. Although the sizes of the gaps cannot be determined by $t_{\mathrm{A}, \mathrm{B}}$ and $Z_{\mathrm{A}, \mathrm{B}}$ alone, they are statistically equal to $Z_{\mathrm{A}, \mathrm{B}}$. Thus, the integration limits of $Z_{\mathrm{A}, \mathrm{B}}$ can be replaced by the observed limits of the rapidity gaps in a given measurement. Noticing that for $\mathrm{A}=\mathrm{B}$, as in pp scattering, the integral of the single-particle inclusive cross section in eq. (2) over the large $Z$ region gives half of the single diffractive cross section, we obtain the total DPE cross section

$\tilde{\sigma}_{\mathrm{DPE}}^{\mathrm{pp}} \approx \frac{\tilde{\sigma}_{\mathrm{SD}}^{\mathrm{pp}}}{4 \sigma_{\mathrm{pp}}^{\mathrm{tot}}} \tilde{\mathrm{SD}}$,

where the tilde indicates the truncation of the cross sections due to the constraints on the sizes of the rapidity gaps. If we impose a minimum size of $\Delta$ for each gap, the integration limits of $Z_{\mathrm{A}, \mathrm{B}}$ are given by the triangle with $Z_{\mathrm{A}, \mathrm{B}} \leqslant \Delta$ and $Z_{\mathrm{A}}+Z_{\mathrm{B}} \leqslant \ln s / s_{\mathrm{o}}$, where $s_{\mathrm{O}}$ is the minimum value of $M_{\mathrm{X}}^{2}$. For $s=1000$ $\mathrm{GeV}^{2}$ and $\Delta=2, \tilde{\sigma}_{\mathrm{SD}}^{\mathrm{pp}}$ is approximately $4 \mathrm{mb}$ and eq. (4) gives $\widetilde{\sigma}_{\mathrm{DPE}}^{\mathrm{pp}} \approx \frac{1}{40} \widetilde{\sigma}_{\mathrm{SD}}^{\mathrm{pp}} \approx 100 \mu \mathrm{b}$. This is a straightforward way to see why the total DPE signal should be about $100 \mu \mathrm{b}$; it is essentially the procedure of ref. [3].

Because of the fact that both the observed gap sizes and the total rapidity range are always finite, there is a finite probability of producing large gaps without the Pomeron exchange. Thus events with two large gaps can arise from single diffractive processes with one of the gaps not generated by the Pomeron. Such non-Pomeron backgrounds are mainly due to the meson-Pomeron exchange (MPE), with one of the Pomerons in the DPE process replaced by a meson trajectory, and possible interference between MPE and DPE. In terms of Mueller regge analysis, the DPE like cross section can be directly related to the triple regge parameters. For small $t$ and $z$, the triple Regge formula for the single-particle inclusive cross section gives [11-13]

$$
\begin{aligned}
& \frac{\mathrm{d}^{2} \sigma_{\mathrm{AB}}^{\mathrm{A}}}{\mathrm{d} t_{\mathrm{A}} \mathrm{d} Z_{\mathrm{A}}}=\sum_{I, J, K=\mathrm{M}, \mathrm{P}} G_{I J K}^{\mathrm{AB} \rightarrow \mathrm{AX}}\left(t_{\mathrm{A}}\right) \\
& \quad \times \exp \left\{\left[\alpha_{I}\left(t_{\mathrm{A}}\right)+\alpha_{J}\left(t_{\mathrm{A}}\right)-2\right] Z_{\mathrm{A}}\right\}\left(M_{\mathrm{X}_{\mathrm{A}}}^{2}\right)^{\alpha_{K}(0)-1}
\end{aligned}
$$

where $\alpha(t)$ represents the regge trajectory. The assumption of short range correlation, which leads to eq. (2) at large rapidity gaps, predicts that the DPE like cross section is given by

$$
\begin{aligned}
& \frac{\mathrm{d}^{4} \sigma_{\mathrm{AB}}^{\mathrm{AB}}}{\mathrm{d} t_{\mathrm{A}} \mathrm{d} Z_{\mathrm{A}} \frac{1}{\mathrm{~d} t_{\mathrm{B}} \mathrm{d} Z_{\mathrm{B}}}} \sum_{\sigma_{\mathrm{AB}}^{\mathrm{tot}}} \sum_{I, J=\mathrm{M}, \mathrm{P}} G_{I J P}^{\mathrm{AB} \rightarrow \mathrm{AX}}\left(t_{\mathrm{A}}\right) \\
& X \exp \left\{\left[\alpha_{I}\left(t_{\mathrm{A}}\right)+\alpha_{J}\left(t_{\mathrm{A}}\right)-2\right] Z_{\mathrm{A}}\right\} \\
& \times\left(M_{\mathrm{X}_{\mathrm{A}}}^{2}\right)^{\alpha} \mathrm{p}^{(0)-1} \frac{\mathrm{d} \sigma_{\mathrm{AB}}^{\mathrm{B}}}{\mathrm{d} t_{\mathrm{B}} \mathrm{d} Z_{\mathrm{B}}}+(\mathrm{A} \leftrightarrow \mathrm{B})\left(1-\delta_{I \mathrm{P}} \delta_{J \mathrm{P}}\right) .
\end{aligned}
$$

From fits to the single particle cross section (5) [12, 13], we know that $G_{\mathrm{MPP}}$ is consistent with zero and therefore the interference effects between DPE and MPE can be neglected. The background is thus given by the integrated results of eqs. (5) and (6) as

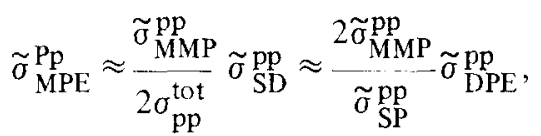

where $\widetilde{\sigma}_{\text {MMP }}$ represents the total MMP contribution to the single-particle inclusive cross section in the given kinematical region. Since $\tilde{\sigma}_{M M P}$ is not directly measurable, we must rely on the triple regge fits to the single-particle cross sections [13-14]. From ref. [14] we obtain $\widetilde{\sigma}_{\mathrm{MMP}}^{\mathrm{pp}} \approx 1.5 \mathrm{mb}$ for $\Delta=2$ and therefore $\tilde{\sigma}_{\mathrm{MPE}}^{\mathrm{pp}} \approx \frac{1}{50} \widetilde{\mathrm{\sigma}} \mathrm{\textrm {SD }}$ or about $80 \mu \mathrm{b}$. The different fits of ref. [13] give somewhat different results but all with the same order of magnitude.

Independent of the triple Regge fits, we can also estimate the background from the inclusive rapidity gap distribution observed in hadronic reactions [14]. Let us denote the probability of producing a nondiffractive gap larger than or equal to $\Delta$ by $P(\Delta)$. Neglecting the interference effects mentioned above, we can then write the single Pomeron exchange (SPE) contribution to the DPE like cross section as

$\tilde{\sigma}_{\mathrm{SPE}}^{\mathrm{pp}} \approx P(\Delta) \tilde{\sigma}_{\mathrm{SD}}^{\mathrm{pp}}$.

Similarly, there is also a nondiffractive contribution given by $[P(\Delta)]^{2}$ times the nondiffractive cross section.

The size of $P(\Delta)$ can be estimated from the observed single-gap inclusive distribution in pp reactions [14] In ref. [14], end gaps have been excluded from the data to minimize the effects of the diffractive (Pomeron) contribution. There may be some remaining Pomeron contribution from the double diffractive processes, in which the gap generated by the Pomeron does not occur at either end. However, since the esti- 
mated double diffractive cross section is small $(\sim 1$ $\mathrm{mb})$ while both the average gap multiplicity $(\sim 10)$ and the nondiffractive cross section are relatively large in the FNAL and ISR energy range, this Pomeron contribution to the inclusive gap distribution should be suppressed by a factor of 300 to 400 compared with the total gap distribution. Furthermore, the fact that the observed gap distributions are rapidly decreasing functions of the gap size [11] also assures us that, after the end gaps are excluded, the Pomeron contribution to the gap distribution is negligible.

From ref. [14], we can see that the probability, $P(\Delta)$, for zero charge transfer gaps $\Delta \geqslant 2$ is about $1 / 70$. Although neutral particles may be present in the gap distributions of ref. [14], we expect that, given no charged particles in two units of rapidity or more, the probability of finding neutral particles in the same region is very small. In particular, this is true if particles are emitted in clusters with two charged particles per cluster on the average, as observed in ref. [14]. For $P(\Delta) \approx 1 / 70$, we obtain $\widetilde{\sigma}_{\mathrm{SPE}}$. $\approx 60 \mu \mathrm{b}$.

Although the triple Regge parameters can not be accurately determined by the fits on the one hand, and we have to assume that the non-Pomeron end gap distribution is approximately the same as the non-end gap distribution on the other. The above two estimates of $\tilde{\sigma}_{\text {SPE }}$ come remarkably close to each other. We conclude that a background signal of $60 \sim 80 \mu \mathrm{b}$ is to be expected. This is comparable to the expected signal for $\widetilde{\sigma}_{\mathrm{DPE}}$.

The important feature of the above analysis is that the background is comparable with the expected signal and that it is possible for the background alone to account for most of the observed cross section. To compare with the ISR data [7], we have to go from our estimates of the total cross section of reaction (1) with two large end gaps to the exclusive cross section with $\mathrm{X}=\pi^{+} \pi^{-}$. The invariant mass of the system $\mathrm{X}$, $M_{\mathrm{X}}$, is given by

$M_{\mathrm{X}}^{2}=s \exp \left\{-\left(Z_{\mathrm{A}}+Z_{\mathrm{B}}\right)\right\}$.

In the FNAL and ISR energy range, $100 \mathrm{GeV}^{2} \leqslant s$ $\leqslant 3000 \mathrm{GeV}^{2}, M_{\mathrm{X}}$ can be at most several GeV for $\Delta$ $=2$ and its average value is even smaller, perhaps 1 $\mathrm{GeV}$ or less [3]. With a small $M_{\mathrm{X}}$, the average multi. plicity of $\mathrm{X}$ should be very low and therefore $\mathrm{X}=\pi^{+} \pi^{-}$ should account for a sizeable fraction of the total cross section. The multiplicity distribution of $\mathrm{X}$ in ref. [7] indeed shows such a feature that about $80 \%$ of the DPE like events only have observed charged multiplicities of 2 or less. The existence of neutrals in the central region should decrease the fraction of $\mathrm{X}$ $=\pi^{+} \pi^{-}$exclusive events relative to the total. But even if the exclusive cross section is only as small as $25 \%$ of the total cross section, the observed 15 to $20 \mu \mathrm{b}$ exclusive cross section can only correspond to a total DPE like cross section of 60 to $80 \mu \mathrm{b}$, which is comparable to the background alone.

On the other hand, since the exclusive cross section of ref. [7] corresponds to the events with two fast leading particles and two charged secondaries in the central region balancing the angles of the momentum vectors, it may be different from the exclusive cross section with $\mathrm{pp} \pi^{+} \pi^{-}$satisfying the overall energy momentum conservation. A study of the selection of events and the angular distribution of the charged secondaries lead us to speculate that the total number of inclusive DPE like events may be 5 to 10 times that of the selected exclusive events. If this the case, the total DPE like cross section may be as large as 75 to $200 \mu \mathrm{b}$. Even with such a large uncertainty, only the upper end of this range can accommodate the expected signal plus background.

Our analysis indicates that the observed DPE like cross section may be much smaller than what is expected and that, due to the large background, no conclusive evidence for the existence of the DPE process has yet been established. But, there are sizeable uncer. tainties in both the estimates of the background and the data. We interpret our results as strongly suggesting that one should ask (1) whether the present understanding of the inclusive theory is really adequate to make these estimates, (2) what is the minimum size of the DPE contribution, and (3) what could be the implications of an experimental DPE signal much smaller than what is expected?

Since there are as yet no clear signals of DPE process, the DPE contribution may be much smaller than the theoretical estimates of refs. [2,3] and eq. (2). Such estimates are based on the assumptions that long range correlations are absent and that the PomeronPomeron total cross section shown in fig. $1 \mathrm{a}$ itself is dominated by an effectively factorizable Pomeron. The least dramatic possibility for the failure of eq. (2) is that $M_{\mathrm{X}}$ is too small, while the Pomeron dominance 
is valid only at large $M_{\mathrm{X}}$. Although the Pomeron dominance seems to work reasonably well for the Pomeron-particle cross section even at low values of $M_{\mathrm{X}}$ [15], the behavior of the Pomeron-Pomeron cross section may be quite different. But, the estimated MPE contribution, which requires the Pomeron dominance of the Pomeron-M total cross section may still not be overestimated, since the Pomeron-M cross section should behave like the Pomeron-particle cross section.

The second possibility is that the Pomeron is not a simple factorizable pole and therefore the PomeronPomeron cross section is not factorizable even though the particle-particle and particle-Pomeron cross sections approximately are. In these two cases, the background can still be as large but the DPE signal may become smaller. A third possibility is the gross failure of the application of the short range correlation ideas to the DPE like processes. Although the $t$-dependences of the fast leading particles appears to be uncorrelated, the charged multiplicity distribution in the central region for the DPE like events is quite different from that for the events without large gaps at both ends [7] . (On the other hand, the multiplicity distributions for the diffractive dissociation and nondiffractive processes are similar to each other.) This difference may indicate the presence of long range correlations. In this case, both the signal and the background may be substantially different from the above theoretical estimates.

For many years it has been believed that a DPE would be present due to the exclusive process $\mathrm{P}+\mathrm{P}$ $\rightarrow \pi^{+} \pi^{-}$with $\pi$ exchange, where $\mathrm{P}$ are the $t$-channel Pomerons of fig. 1a. Then factorization of the pion pole gives $\pi \mathrm{N}$ scattering at each side and thus two Pomerons. Even those who feel intuitively that a Pomeron is a shadow of inelastic processes and consequently can only be present once in a given process have not been able to give an analytic argument against the above point. Thus, this process represents in a sense the minimal DPE signal acceptable to present theory without serious consequences. It has been estimated by Henyey and Pumplin [16] to be about $10 \mu \mathrm{b}$, which is not inconsistent with existing numbers. It is not clear why the rest of the total DPE should be so small. It would be appropriate for theorists with definite Pomeron models to make estimates of the DPE signal in their theory.
To clarify the picture of the recurrence of the Pomeron, we need both a better theoretical estimate of the cross sections and more definitive experimental tests. For the latter, we need to discuss some more detailed features of the DPE and SPE contributions. For a fixed minimum gap size $\Delta$, both contributions should approximately scale. In fact, since both depend on $\sigma_{\mathrm{SD}}$, which is increasing with $s$ in the FNAL and ISR energy range, they should also increase slowly. But such an increase may be offset by the decrease of some entirely non-Pomeron contribution and the cross section may be constant, or even decreasing with $s$, as observed in refs. [4-7]. The azimuthal correlation of the leading particles [10] can only test whether the Pomeron-Pomeron or meson-Pomeron cross section is dominated by a simple pole but can not test whether the DPE or SPE process is dominant. The factorization property of the $t_{\mathrm{A}, \mathrm{B}}$-dependence can in principle determine whether the DPE contribution dominates, but the MPE contribution only causes a small deviation from factorization over the limited range of $t_{\mathrm{A}, \mathrm{B}}$ measured in ref. [7].

Probably, the most decisive test for DPE processes is the behavior of the gap size $(\Delta)$ dependence of the cross sections. The DPE contribution to the differential cross section is independent of $Z_{\mathrm{A}, \mathrm{B}}$ and the total cross changes slowly with the available kinematical range, $\sigma_{\mathrm{DPE}} \propto\left(\ln s / s_{\mathrm{O}}-\Delta\right)^{2}$. On the other hand, the MPE contribution decreases exponentially in $Z_{\mathrm{A}, \mathrm{B}}$ and the total cross section behaves like $\exp \left\{\left[2 \alpha_{M}(0)\right.\right.$ $-2)] \Delta\}\left(\ln s / s_{\mathrm{O}}-\Delta\right)^{2}$. Thus a measurement of the $Z_{\mathrm{A}, \mathrm{B}}$-dependence of the differential cross section or the $\Delta$-dependence of the total cross section should be able to determine the size of the DPE contribution. In particular, we can see whether the total cross section divided by the phase space $\left(\ln s / s_{0}-\Delta\right)^{2}$ has a constant term, which is the DPE contribution. If the estimate of $\tilde{\sigma}_{\mathrm{MPE}}$ is correct, namely, $\sigma_{\mathrm{SPE}} \approx \widetilde{\sigma}_{\mathrm{MPE}}$, the leading meson trajectories with $\alpha_{M}(0)=1 / 2$ are the leading contributions to $\sigma_{\mathrm{SPE}}$ and we should have $\widetilde{\sigma}_{\mathrm{SPE}} \propto \mathrm{e}^{-\Delta}\left(\ln s / s_{\mathrm{O}}-\Delta\right)^{2}$. Varying from $\Delta=2$ to $\Delta$ $=3$ should make $\widetilde{\sigma}_{\mathrm{DPE}} / \widetilde{\sigma}_{\mathrm{SPE}}$ increase by a factor of three.

If a clear signal of the DPE processes can be established, one may further ask whether the PomeronPomeron cross section can be dominated by an approximately factorizable Pomeron at large $M_{\mathrm{X}}$. Unfortunately, large $\Delta$ and large $M_{\mathrm{X}}$ cannot be simultaneously 
achieved at the presently available energies but may be at ISABELLE energies in the future.

We have tried to emphasize the following points:

1) The existence and size of a double Pomeron exchange contribution are important aspects of our entire view of the nature of the Pomeron.

2) The experimental signal appears to be comparable with a "background" from single Pomeron exchange and a sizeable second rapidity gap of small but finite probability. Thus a true double Pomeron signal has not yet been established.

3) Considerable clarification of the situation could come from a study of the behavior of the cross section with changes in the rapidity gap (at fixed $s$ and large gap size). A true double Pomeron signal would be constant as the gap size increased (apart from phase space) while the single Pomeron background will fall exponentially with increasing gap size.

We appreciate stimulating discussions with G.F. Chew, D.M. Chew, J.W. Dash, F. Henyey, J. Pumplin, H.I. Miettinen, and R.G. Roberts.

\section{References}

[1] J. Finkelstein and K. Kajantie, Nuovo Cimento 56A (1968) 658.

[2] R. Shankar, Nucl. Phys. B63 (1974) 168.
[3] D.M. Chew and G.F. Chew, Phys. Lett. 53B (1974) 191.

[4] D.M. Chew et al., preprint LBL-2106 (1973).

[5] M. Derrick et al., Phys. Rev. Lett. 32 (1974) 80.

[6] E.L. Berger et al., CERN preprint D.Ph. TI/PHYS 74-26, Proc. 17th Intern. Conf. on High Energy Physics, London, July 1974.

[7] L. Baksay et al., CERN preprint, submitted to the EPS Intern. Conf. on High-Energy Physics, Palermo, June (1975).

[8] Some of these problems are also studied by H.I. Miettinen, talk given at the EPS Intern. Conf. on High Energy Physics, Palermo, June (1975) and R.G. Roberts, private communication. Roberts has independently done estimates which are consistent with ours for the quantities we discuss.

[9] A.H. Mueller, Phys. Rev. D4 (1971) 150.

[10] D.Z. Freedom, C.E. Jones, F.E. Low and J.E. Young, Phys. Rev. Lett. 26 (1971) 1197;

C. Jen, K. Kang, P. Shen and C.-I. Tan, Phys. Rev. Lett. 27 (1971) 458.

[11] L. Caneschi and A. Pignotti, Phys. Rev. Lett. 22 (1969) 1279;

D. Silverman and C.-I. Tan, Phys. Rev. D2 (1970) 233;

C.E. DeTar et al., Phys. Rev. Lett. 26 (1971) 675.

[12] R.D. Field and G.C. Fox, Nucl. Phys. B80 (1974) 367.

[13] D.P. Roy and R.G. Roberts, Nucl. Phys. B77 (1974) 240.

[14] P. Pirilä, G.H. Thomas and C. Quigg, Phys. Rev. D12 (1975) 92.

[15] J.W. Dash, Phys. Rev. D9 (1974) 200; N.F. Bali and J.W. Dash, Phys. Lett. 37B (1971) 292; Also see the arguments given in ref. [3] for the Pomeron dominance of the Pomeron-Pomeron cross section.

[16] F.S. Henyey and J. Pumplin, private communication. 